\section{Laterality defects in conjoined twins}

SIR - Gene expression in twin chick embryos provides insight into the cause of the laterality defects in human conjoined twins ${ }^{1-3}$. We surveyed 167 pairs of conjoined twins obtained locally and from the literature (details and references available from D. J. R. on request; e-mail: robertsd@helix.mgh.harvard.edu). The propensity towards laterality defects depends on the orientation of the conjoined twins ( $a$ in the figure). In almost half of the cases (33 of 69) where the twins were joined obliquely at the chest and/or abdomen (thoracopagi) or laterally at the chest (dicephali), one of the twins had reversal in heart situs; however none of the twins (of 98 studied) joined only at the head (craniopagi) or pelvis (ischiopagi) exhibited laterality defects. Strikingly (as noted in refs 1-3), when laterality defects are present, they occur most frequently in the twin on the right side in dicephalus $(86 \%)$ and thoracopagus $(71 \%)$ twins.

In the chick, a cascade of secreted signals during gastrulation regulates leftright asymmetry ${ }^{4}$. Activin, on the right side of the primitive streak, represses expression of the gene Sonic hedgehog (Shh) on the right. The remaining expression of $S h h$ on the left is responsible for inducing nodal on the left, ultimately specifying heart situs. When twins arise from two parallel primitive streaks, there is the possibility of the activin produced on the right side of the left embryo inhibiting the expression of $S h h$ in the left side of the right embryo ( $c$ in the figure). This would result in a normal left embryo, but the right embryo would have no expression of $S h h$ in the node, and therefore no expression of nodal, and hence would have random heart situs ${ }^{4}$. Consistent with this hypothesis, we find that in spontaneous twin chick embryos with parallel primitive streaks, Shh $(e$ in the figure) and nodal $(f)$ are always expressed normally on the left of the left-side embryo, but never observed on either side of the right-side embryo.

If the twin primitive streaks form at an angle rather than being completely parallel, the two nodes become closer together as the streaks elongate. In some instances, the repression of $S h h$ by activin will proceed normally because the streaks will initially be too far apart for the signals to diffuse. However, as the embryos elongate anteriorly, the Shh product (Shh) on the left side of the right embryo could cause nodal to be induced on the right side of the left embryo. In this case, the right embryo will be normal, but the left embryo will have double-sided nodal expression, leading to
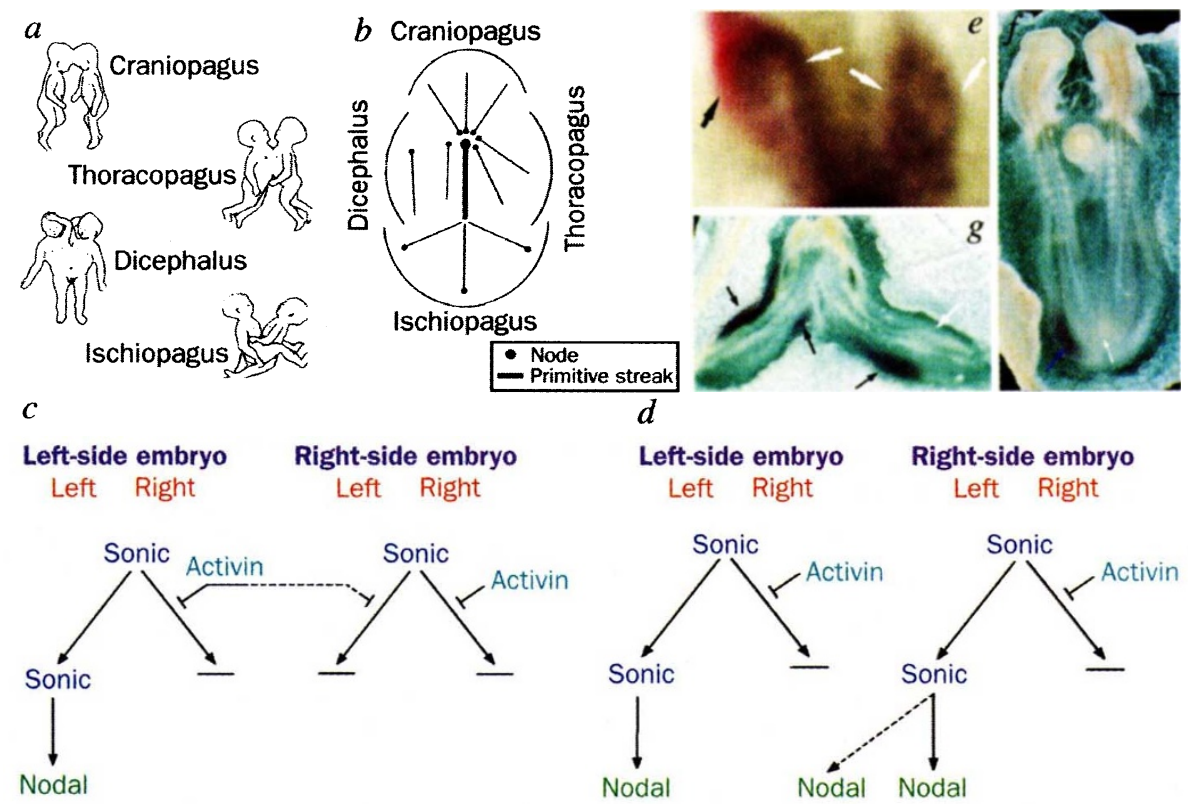

Left-side embryo

Right-side embryo

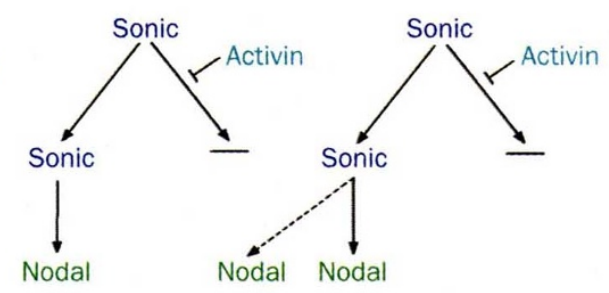

Four classes of human conjoined twins (a, modified from ref. 11), have been suggested to arise from ectopic streaks ( $b$, modified from refs 5-7). Parallel twin streaks $(c)$ potentially allow activin cross-signalling to the right embryo. Such embryos hybridized to a Shh probe show normal Shh expression (magenta stain, black arrow in part e) in the left embryo, and a lack of expression (white arrows) in the right at embryonic stage 4-5 (2 of 2 cases examined). When hybridized to a nodal probe, such embryos show normal nodal expression (blue arrow, bottom in part $f$ ) in the left embryo and a lack of expression (white arrow, bottom) of nodal in the right embryo, which can result in inverted heart situs (black arrow) (2 of 2 cases examined). Oblique twin streaks that only become close as they extend (d) show normal nodal expression ( $g$; black arrows, compare with white arrows) in the right embryo and double nodal expression (black arrows) in the left embryo ( 4 of 4 cases examined). The study was made possible by the assistance and cooperation of J. Tabin, S. Van Praagh, R. Van Praagh, M.-N. Westgate, P. lyangar, J. Westerdahl, the Active Malformations Surveillance Program, the Departments of Pathology at Brigham and Women's Hospital and Boston's Children's Hospital, the Hensel family, and the many additional families contacted for information on family members, conjoined and separated.

randomization of its situs ( $d$ in the figure). This is precisely what we observed in obliquely oriented spontaneous twin embryos $(g)$.

There have been several models of the origins of various classes of conjoined twins based on the relative orientation of their primitive streaks at gastrulation $(b$ in the figure; refs 5-7). In craniopagi and ischiopagi twins, the primitive streaks are end-to-end, and signalling molecules to the side of one would not be expected to affect the other. However, dicephali and thoracopagi twins arise from streaks positioned adjacent to each other, potentially allowing cross-signalling. When primitive streaks are not completely parallel, which embryo has laterality defects will depend on their relative angle and distance apart. One would expect the left-side twin to have laterality defects in a higher proportion of thoracopagus conjoined twins than in dicephalus twins, as they result from primitive streaks oriented at a more oblique angle. This is indeed what is observed.

Activin, Shh and nodal normally act only on the side of the embryo where they are expressed, yet can apparently signal at a longer range between embryos. This suggests the presence of a barrier in the midline of the embryo to confine each signal to its appropriate side. There is evidence that, in the amphibian Xenopus, midline structures do indeed facilitate regulation of situs ${ }^{8}$.

Although there are differences in the regulation of left-right asymmetry in birds and mammals, some aspects such as nodal expression are conserved ${ }^{9,10}$. As such, the chick model provides the first coherent explanation for the laterality defects observed in conjoined twins.

Michael Levin

Drucilla J. Roberts

Lewis B. Holmes

Cliff Tabin

Harvard Medical School,

Boston, Massachusetts 02115, USA

1. Aird, I. Brit. Med. J. 1, 1313-1315 (1959).

2. Cuniff, C. et al. Am. J. Med. Genet. 31, 669-677 (1988).

3. Torgersen, J. Arch. Pathol. 47, 566-593 1949).

4. Levin, M., Johnson, R., Stern, C., Kuehn, M. \& Tabin, C. Cell 82, 1-20 (1995).

5. Spencer, R. Teratology 45, 591-602 (1992).

6. Spencer, R. Teratology 52, 346-356 (1995)

7. Kapur, R. P., Jack, R. M. \& Seiber, J. R. Am. J. Med. Genet. 52, 188-195 (1994).

8. Danos, M. C. \& Yost, H. J. Development 121, 1467-1474 (1995)

9. Lowe, L. et al. Nature 381, 158-161 (1996).

10. Collignon, J., Varle, I. \& Robertson, E. Nature $\mathbf{3 8 1}$, 155-158 (1996)

11. Arey, L. B. Developmental Anatomy (Saunders, Philadelphia, 1965) 